\title{
A new challenge for the world: the eradication of polio
}

\author{
Ángela Gentile, M.D. ${ }^{a}$ and Héctor Abate, M.D. ${ }^{b}$
}

\begin{abstract}
Poliovirusinfects $100 \%$ of susceptibleindividuals and causes acute flaccid paralysis in one out of 200 infections. Type 1 causes epidemic poliomyelitis; type 2 has been eradicated worldwide; and type 3 is close to being eradicated. In this region, the last case of wild poliovirus occurred in Peru in 1991. There are still two endemic countries: Afghanistan and Pakistan, but countries where there is no circulation of the wild poliovirus have also reported imported cases of polio. In May 2012, the World Health Assembly declared the polio eradication a programmatic emergency forglobal publichealth and, as a result, developed the Polio Eradication and EndgameStrategic Plan 2013-2018. The Plan has four objectives: 1) Detect and interrupt all poliovirus transmission and maintain surveillance of acute flaccid paralysis in children $<15$ years. 2) Strengthen immunization systems and withdraw oral polio vaccine by the first trimester of 2016. Replace the trivalent oral polio vaccine with the bivalent oral vaccine, containing serotypes 1 and 3 , and introduce the inactivated polio vaccine in all immunization schedules to maintain immunity against poliovirus type 2. 3) Contain poliovirus and certify interruption of transmission. 4) Plan the exploitation of the fight against polio and its impact on public health. The plan is expected to reach its goals by 2018; all use of the oral polio vaccine will be interrupted thereafter. Change in immunization schedules will require pediatricians to provide advice and guidance to families depending on the varied situations of everyday practice.
\end{abstract}

Keywords:poliomyelitis, eradication, immunization.

http:/ / dx.doi.org/10.5546/aap.2016.eng.557

Poliovirus belongs to the Enterovirus genus of the Picornaviridae family. There are three types: type 1 , which most commonly causes epidemic poliomyelitis; type 2 , which has been eradicated worldwide; and type 3, which has geographically limited circulation and is close to being eradicated. ${ }^{1}$

Poliovirus is highly contagious. It generally infects $100 \%$ of susceptible individuals, especially children younger than 5 years old. Asymmetric acute flaccid paralysis (AFP), with areflexia in the affected extremity but no sensory deficit, occurs only in 1 out of 200 primary infections. ${ }^{2}$ The paralytic polio case/infection ratio varies depending on poliovirus type: type $1,1 / 190$ infections; type 2, 1/1886; and type 3, $1 / 1149$. The main route of transmission is fecal-oral transmission, especially in developing countries due to poor hygienic and sanitary conditions. The incubation period (from infection to paralysis onset) usually ranges from 7 to 21 days (3-35 days), and the virus spreads rapidly. Virus excretion is greater before the onset of paralysis and during the first two weeks, when it is present in the throat and in high concentration in feces. In some people, excretion may last up to two months. ${ }^{3}$

Poliomyelitis was always supposed to be an eradicable disease because it has no animal reservoir. The implementation of an effective prevention tool, the oral and the inactivated polio vaccines, dramatically reduced paralytic poliomyelitis and became one of the most successful public health programs worldwide. ${ }^{4}$ Besides, the virus does not easily survive in the environment, making it vulnerable. However, given that the infection is highly symptomatic $(72 \%)$ and that there are other diseases with similar symptoms, other difficulties have been identified.

The last case of wild poliovirus (WPV) in this region was detected in Peru in 1991. In 1994, the Global Commission for the Certification of the Eradication of Poliomyelitis reviewed the evidence available in every country or territory and concluded that endemic WPV circulation had stopped across the continent, making the Americas the first region to achieve this goal worldwide. ${ }^{5}$ 
In the following years, other three regions were certified to be polio-free: the Western Pacific region in 2000; the European region in June 2002; and the South-East Asia region (including India) in March 2014. At present, there are only two endemic countries: Afghanistan and Pakistan. However, some non-endemic countries have reported, after several years of no WPV circulation, imported cases of polio that resulted in secondary cases. ${ }^{6,7}$

In May 2012, the World Health Assembly declared the polio eradication a programmatic emergency for global public health and called to implement a comprehensive endgame strategy for polio eradication. As a result, the Polio Eradication and Endgame Strategic Plan 2013-2018 was developed. ${ }^{8,9}$

The plan offers a comprehensive approach to fully achieve eradication, including the elimination of all polio diseases, either caused by WPV or related to the vaccine. This strategic plan, which will be simultaneously implemented worldwide, has four objectives:

\section{Detect and interrupt all poliovirus transmission}

The strategy of the epidemiological surveillance system is to report and study all cases of AFP in children younger than 15 years of age, as usual, in order to detect WPV circulation, or demonstrate its absence, or to prove that surveillance meets the performance requirements to obtain the certification.

AFP surveillance will continue to be the priority mechanism to detect poliovirus circulation. An adequate epidemiological surveillance, with goodquality data, serves as guidance for immunization activities. For this reason, compliance with each country's surveillance indicators is critical. ${ }^{10}$

The two key indicators of surveillance performance are:

a. Non-poliomyelitis AFP in children $<15$ years: minimum goal $\geq 1 / 100,000$ in the $<15$-yearold population; or, in endemic countries with outbreaks or at risk for imported polio cases, $\geq 2 / 100,000$ in the $<15$-year-old population. The rate of case reporting has been achieved in the region since 1988.

b. Adequate stool samples: collected at least 24 hours apart and within 14 days of the onset of paralysis. Minimum goal: $\geq 80 \%$. The percentage of AFP cases with adequate samples and testing within 48 hours were not reached in the past recent years, except in some countries of the region (Figures 1, 2, 3).

\section{Strengthen immunization systems and withdraw oral polio vaccine (OPV)}

Vaccines containing live attenuated viruses are highly effective against WPV but, in rare cases, may cause AFP by means of two different mechanisms: ${ }^{11,12}$

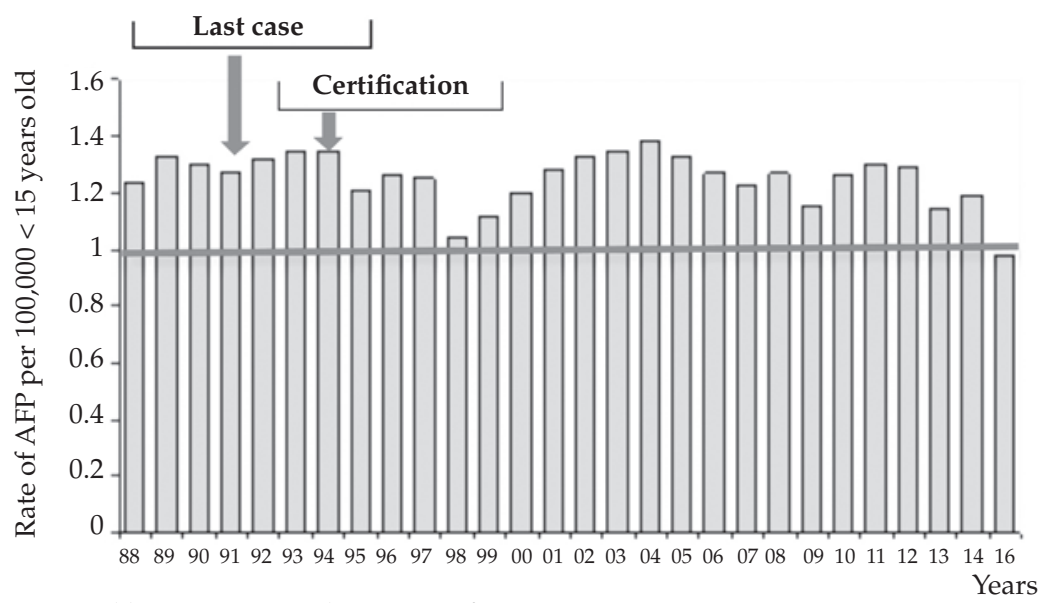

Source: ISIS, PESS or reported by countries to the FGL-IM/PAHO.

AFP: acute flaccid paralysis. PESS: Polio Eradication Surveillance System. ISIS: Integrated Surveillance and Informatics Services. 
1. Reacquisition of neurovirulence: live attenuated viruses contained in the OPV may, by means of prolonged replication in an immunosuppressed individual or a community with a low immunization coverage, reacquire the neurovirulence and transmissibility typical of WPV. Such viruses may cause paralytic poliomyelitis cases or outbreaks. Genetic divergence of type 1 and type 3 poliomyelitis should be higher than $1 \%$ while that of type 2 should be higher than $0.6 \%$.
Vaccine-derived polioviruses are sub-divided into three categories:

a. Circulating vaccine-derived poliovirus (cVDPV): when there is evidence of person-to-person transmission in the community. cVDPV was first described in 2000 during an outbreak on the island of Hispaniola (Haiti and Dominican Republic). Recent experience indicates that a low immunization coverage is a major risk factor for cVDPV outbreaks. These

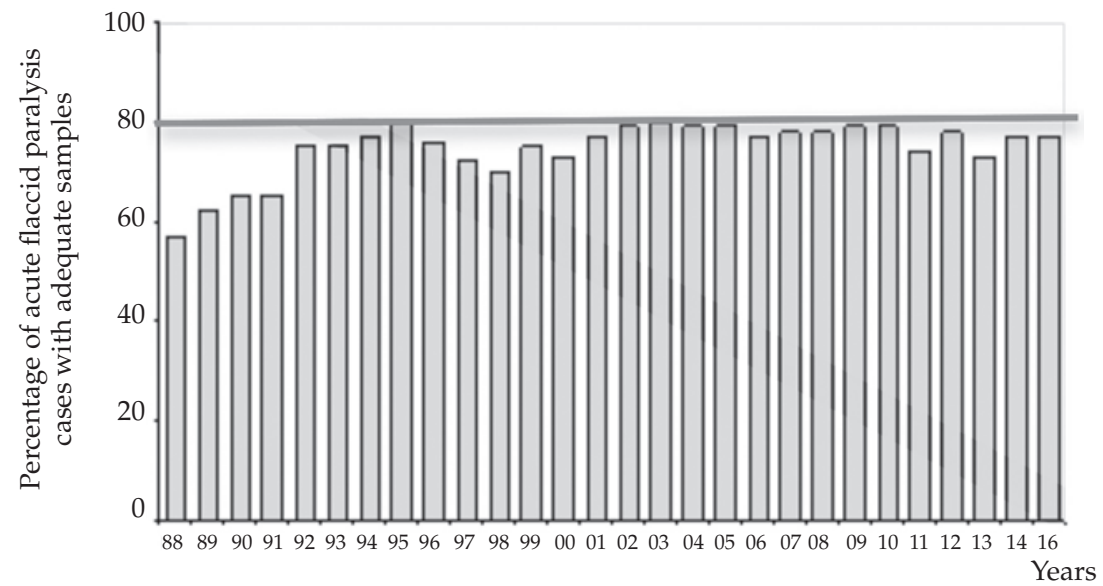

Source: ISIS, PESS or reported by countries to the FGL-IM/PAHO.

AFP: acute flaccid paralysis. PESS: Polio Eradication Surveillance System. ISIS: Integrated Surveillance and Informatics Services.

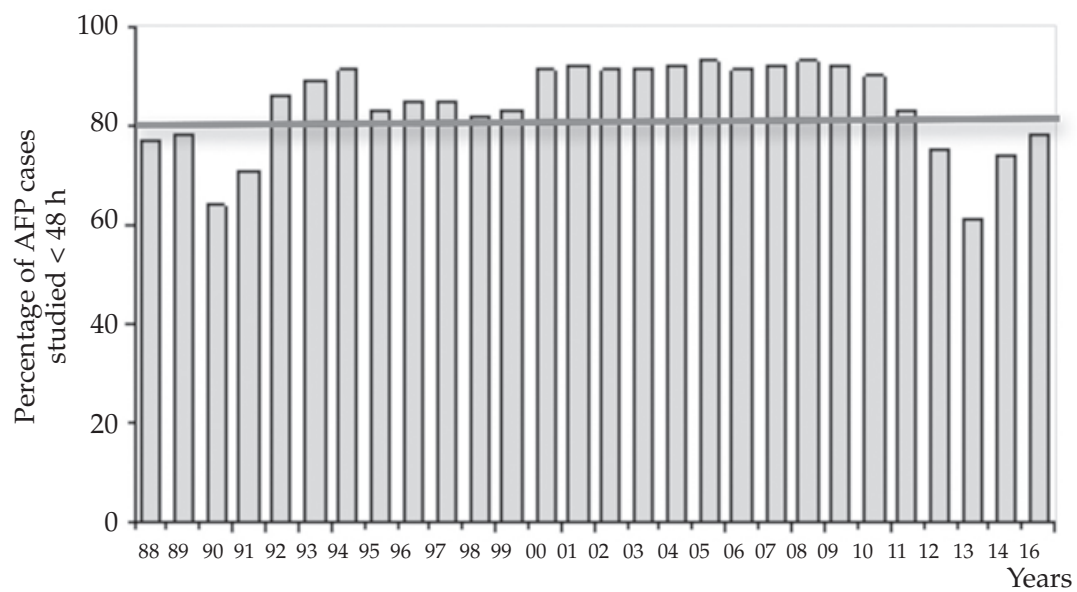

Source: ISIS, PESS or reported by countries to the FGL-IM/PAHO. AFP: acute flaccid paralysis. 
viruses are capable of becoming endemic, as observed in the island of Hispaniola, Nigeria, and Egypt. ${ }^{13}$

b. Immunodeficiency-related vaccine-derived poliovirus (iVDPV): this mainly occurs in cases of B-cell immunodeficiency. ${ }^{1}$ Virus excretion in people with immune system impairment is prolonged; cases of virus excretion for 10 or more years have been reported.

c. Ambiguous vaccine-derived poliovirus: either isolated from people with no known immunodeficiency, or isolated from sewage whose ultimate source is unknown.

2. Mutation associated with neurovirulence: this mechanism is the cause of vaccine-associated paralytic polio (VAPP). This is an uncommon event, associated with the administration of the OPV, and caused by a mutated virus that regained its neurovirulence. In the region of the Americas, the estimated risk of VAPP in a vaccine receptor or a close contact is $1 / 7.68$ million doses administered (95\% confidence interval of 1/6.73-1/8.95 million doses administered). Worldwide, there are 250-500 estimated VAPP cases per year; out of these, nearly $40 \%$ are caused by type 2 contained in the trivalent oral polio vaccine (tOPV). ${ }^{11,12,13}$

Steps are as follows:

a. At the end of 2015 and up to the first trimester of 2016, always before the switch or at the same time, it is necessary to introduce at least one dose of the inactivated polio vaccine (IPV) in all routine immunization schedules. That is to say, before switching from the tOPV to the bivalent oral polio vaccine (bOPV), which contains type 1 and type 3 poliovirus. ${ }^{14}$ At this stage, studies conducted in Latin America on bOPV immunogenicity following one or more IPV doses have been crucial. A study conducted by O'Ryan et al. investigated seroconversion rates against type 1 and type 3 poliovirus, and the proportion of children with protective antibodies; also sequential regimens containing IPV and bOPV were compared to those containing only IPV. It was established that one or two bOPV doses, following IPV regimens, did not provide different titers or percentages of protected children. In a study of immunogenicity of one versus two versus three IPV doses in a sequential regimen with bOPV, it was observed that two or three IPV doses provided a better seroconversion, and that one dose provided protection and induced immunity if followed by exposure to the vaccine virus. The study of an IPV effect on poliovirus excretion in stools showed a greater impact on excretion when the bOPV was administered to a child who had already received the IPV than when three IPV doses were administered without the bOPV. ${ }^{15}$

These studies allow health authorities certain flexibility when choosing an immunization schedule with one or two IPV doses followed by the bOPV.

b. In the second fortnight of April 2016, the tOPV should be switched to the bOPV (which does not contain type 2 poliovirus) in routine immunization schedules and in polio immunization campaigns. ${ }^{14}$

c. Eventual withdrawal of all OPV should be planned. ${ }^{14}$

The introduction of the IPV is key for the eradication and endgame plan and for the worldwide preparation towards risk reduction in association with the withdrawal of the type 2 component in the OPV. It is critical to introduce at least one IPV dose by the end of 2015. In Argentina, two doses will be introduced as of May 1 ${ }^{\text {st }}, 2016$.

The main role of the IPV will be to maintain immunity against type 2 poliovirus while the type 2 poliovirus component in the OPV is withdrawn from the market worldwide. More specifically, IPV introduction is necessary for the following reasons:

- To reduce risks. Once type $2 \mathrm{OPV}$ is withdrawn from the market globally, there will be an unprecedented accumulation of individuals susceptible to this type of poliovirus. Using the IPV will help to maintain immunity and prevent type 2 poliovirus emergency, if this is reintroduced. Therefore, a region where the population has gained immunity through the IPV would have a lower risk for re-emergence or reintroduction of the type 2 WPV or vaccine-derived WPV.

- To interrupt transmission in case of an outbreak. If it is necessary to use the type 2 monovalent oral polio vaccine (mOPV) to control an outbreak, it will be easier to reach immunity levels required to interrupt transmission in a population that has already received the IPV. 
Therefore, introducing the IPV may facilitate the management of future outbreaks.

Although type 2 WPV has been eradicated, and this has been officially announced by members of the Global Commission on September $20^{\text {th }}, 2015,{ }^{16}$ vaccine-related type 2 viruses continue causing most cVDPV outbreaks and VAPP cases. Therefore, type 2 OPV brings more risks than benefits, and hinders global efforts to eradicate poliomyelitis. For this reason, the tOPV will be replaced with the bOPV, which will continue to fight type 1 and type 3 poliovirus. Once these types are eradicated, the bOPV will also be withdrawn from the market.

The World Health Organization's Strategic Advisory Group of Experts (SAGE) on Immunization has called for the withdrawal of the tOPV, which contains type 2 poliovirus, during 2016. This will set the stage for ending bOPV use by 2019-2020, once worldwide eradication has been achieved. As a risk mitigation measure, the SAGE recommends that before switching from the tOPV to the bOPV, every country currently using the OPV in their routine immunization schedules should introduce at least one IPV dose in their routine schedules, i.e., by the end of 2015.

Countries with lower risk levels will change to the IPV after the planned switch due to a low IPV availability. Argentina, which is in the latter group, will switch to the IPV and replace the tOPV with the bOPV at the same time.

Throughout this process it is critical to maintain adequate vaccine coverage levels: at least a $95 \%$ global coverage and a higher than $90 \%$ coverage in each district or municipality.

\section{Contain poliovirus and certify interruption of transmission}

The $68^{\text {th }}$ World Health Assembly calls countries to adequately confine type $2 \mathrm{WPV}$ in essential facilities before the end of 2015 and type 2 Sabin poliovirus strains within three months as of the global withdrawal of the type 2 component in the OPV, in April 2016 (WHA68.3 resolution dated May $26^{\text {th }}, 2015$ ).

The objectives include reducing the risk of reintroduction to a minimum following eradication and containment of WPV, the sequential interruption of routine OPV use, and the introduction of the specific-type IPV by reducing the number of laboratories where polioviruses are contained and that play critical roles in vaccine production, diagnosis, referral and research, in compliance with all necessary warranties.
A national survey was conducted among laboratories and laboratory facilities and an inventory was made of infectious or potentially infectious WPV or bOPV materials. Non-essential facilities agreed to destroy all unnecessary or potentially infectious WPV or bOPV materials. Essential facilities retaining polioviruses will be defined by health authorities and be certified nationwide to ensure adequate containment.

\section{Plan the exploitation of the fight against polio and its impact on public health}

The region may help with global documentation by sharing its experience and lessons learned. The goal is to establish the impact caused by the Expanded Programme on Immunization (EPI) and polio eradication on the Americas' health systems, specifically community involvement and management strengthening at each level of public health. ${ }^{17}$

In this process, pediatricians' role is important, especially in terms of epidemiological surveillance and immunization actions. Immediately reporting all AFP cases will allow to monitor this process and quickly detect all cases of patients with cVDPV or outbreaks.

Change in immunization schedules will require pediatricians to provide advice and guidance to families depending on the varied situations of everyday practice. This is a historical time, just like when our predecessors witnessed the eradication of smallpox. Every country worldwide, including Argentina, faces the same challenges: coordinating sectors for the provision of the IPV, carrying out a massive and simultaneous destruction of the tOPV and replacing it with the bOPV, maintaining an adequate epidemiological surveillance with goodquality indicators and, most importantly, reaching commitment and vocation for union among everyone involved in this challenge.

\section{REFERENCES}

1. American Academy of Pediatrics. Infecciones por el virus de la Polio. In Kimberlin DW, Brady MT, Jackson MA, Long SS, eds. Red Book: Informe 2015 del Comité sobre Enfermedades Infecciosas. 30.a ed. Elk Grove Village, IL: American Academy of Pediatrics; 2015. Pp.644-50.

2. Sutter RW, Kew OM, Cochi SL, Aylward B. Poliovirus vaccine-live. In Plotkin SA, Orenstein WA, Offit PA, eds. Vaccines. 6th ed. Edinburgh: Elsevier-Saunders; 2013. Pp.598-645.

3. Nathanson N, Kew OM. From emergence to eradication: the epidemiology of poliomyelitis deconstructed. Am J Epidemiol 2010;172(11):1213-29.

4. Center for Disease Control and Prevention (CDC). Progress towardsinterrupting wild poliovirus transmission worldwide, 2009. MMWR Morb Mortal Wkly Rep 2010;59(18):545-50. 
5. Centers for Disease Control and Prevention (CDC). International Notes Certification of Poliomyelitis Eradication the Americas, 1994. MMWR Morb Mortal Wkly Rep 1994;43(39):720-2.

6. Sánchez AL. La erradicación de la poliomielitis en la Región Europea de la Organización Mundial de la Salud. Rev Esp Salud Pública 2013;87(5):507-16.

7. Obregón R, Chitnis K, Morry C, Feek W, et al. Achieving polio eradication: a review of health communication evidence and lessons learned in India and Pakistan. Bull World Health Organ 2009;87(8):624-30.

8. World Health Organization. PolioEradication and Endgame Strategic Plan 2013-2018. Geneva, 2013. [Accessed on: July $24^{\text {th }}, 2015$. Available at: http:/ / www.polioeradication.org/ Resourcelibrary/Strategyandwork.aspx.

9. Aylward B, Tangermann R. The global polio eradication initiative: lessons learned and prospects for success. Vaccine 2011;29(Suppl 4):D80-5.

10. Organización Mundial delaSalud. Vigilancia y Red Mundial de Laboratorios Antipoliomieliticos. Vigilancia para detectar los casos de Poliomielitis. Ginebra, 2016. [Accessed on: April 5 ${ }^{\text {th }}$ 2016. Available at: http://www.who.int/topics/ poliomyelitis/surveillance/es/.
11. Platt LR, Estivariz CF, Sutter RW. Vaccine-associated paralytic poliomyelitis: a review of the epidemiology and estimation of the global burden. J Infect Dis 2014;210(Suppl 1):S380-9.

12. Diop OM, Burns CC, Sutter RW, Wassilak SG, et al. Update on Vaccine-Derived Polioviruses - Worldwide, January 2014-March 2015. MMWR Morb Mortal Wkly Rep 2015;64(23):640-6.

13. Kew OM, Wright PF, Agol VI, Delpeyroux F, et al. Circulating vaccine-derived polioviruses: current state of knowledge. Bull World Health Organ 2004;82(1):16-23.

14. World Health Organization. Polio vaccines: WHO position paper, January 2014-recommendations. Vaccine 2014;32(33):4117-8.

15. O'Ryan M, Bandyopadhyay AS, Villena R, Espinoza M, etal. Inactivated poliovirus vaccine given alone or in a sequential schedule with bivalent oral poliovirus vaccine in Chilean infants: a randomised, controlled, open-label, phase 4, noninferiority study. Lancet Infect Dis 2015;15(11):1273-82.

16. World Health Organization. Weekly epidemiological record. Geneva, 2015. Report Nro 50, 2015, 90.

17. Orenstein WO. Eradicating polio: how the world's pediatricians can help stop this crippling illness forever. Pediatrics 2015;135(1):196-202. 